\title{
Utilisation de beurres en cosmétique
}

Olivier PAQUATTE

Laserson, BP 57, PA Sudessor, 91151 Etampes cedex

\section{Introduction}

II y a aujourd'hui une forte demande des consommatrices pour de nouvelles textures, pour des produits naturels, exotiques ou dérivés de l'alimentaire. Les beurres sont des ingrédients qui s'inscrivent parfaitement bien dans ces tendances. On trouve deux types de beurres. Premièrement, les beurres naturels qui sont directement extraits de graines ou noyaux de plantes par pressage ou extraction par des solvants. Ils subissent ensuite un traitement visant à les purifier (filtration ou traitement à la vapeur). Deuxièmement, les beurres «non naturels » qui sont des mélanges d'huiles hydrogénées qui confèrent au produit la texture typique des beurres, avec d'autres huiles. Dans cet article, nous nous concentrerons sur les beurres naturels qui s'inscrivent totalement dans la tendance pour les produits naturels.

\section{Étude sur les produits cosmétiques contenant des beurres naturels}

Nous avons réalisé une étude sur les produits cosmétiques lancés en Europe avec des beurres naturels depuis 2006 (figure 1). Les pays étudiés sont la France, I'Allemagne, I'Angleterre, I'Espagne et I'Italie. L'étude montre qu'il y a une forte augmentation des lancements de produits contenant des beurres naturels en Europe. II y avait trois fois plus de lancements en Europe en 2008 qu'en 2006.

Les beurres naturels sont trouvés principalement dans les produits de soin et de maquillage (figure 2). Les lancements de beurres naturels se concentrent dans les produits pour le visage, le cou, le corps, les lèvres et le contour des yeux (figure 3). Tous les grands acteurs de la cosmétique européenne formulent des beurres natu-

\begin{abstract}
There is a clear consumer need for new textures and for natural and food inspired ingredients in cosmetics. Natural butters are fully in line with these trends. As a result, more and more products containing butters are launched on the European cosmetic market. They are found mainly in skincare and colour cosmetic products. Butters are used as emollients, texturing agents or to perfume finished products. Depending on their composition, their sensorial profiles will vary. Butters are also very effective marketing tools.
\end{abstract}

Key words: butters, cosmetics, european cosmetic market rels dans leurs produits : L'Oréal, Chanel, Estée Lauder, Johnson \& Johnson, Procter \& Gamble, etc. On trouve des produits contenant des beurres dans tous les grands canaux de distribution : le sélectif (LVMH), la grande distribution (Beiersdorf), la pharmacie (Pierre Fabre), la vente par correspondance (Yves Rocher).
Formulation des beurres en cosmétique

Les beurres sont généralement ajoutés dans la phase huileuse chauffée entre 40 et $70{ }^{\circ} \mathrm{C}$. Ils peuvent également être fondus et utilisés directement comme phase huileuse dans les émulsions de type " huile dans eau ». II faut

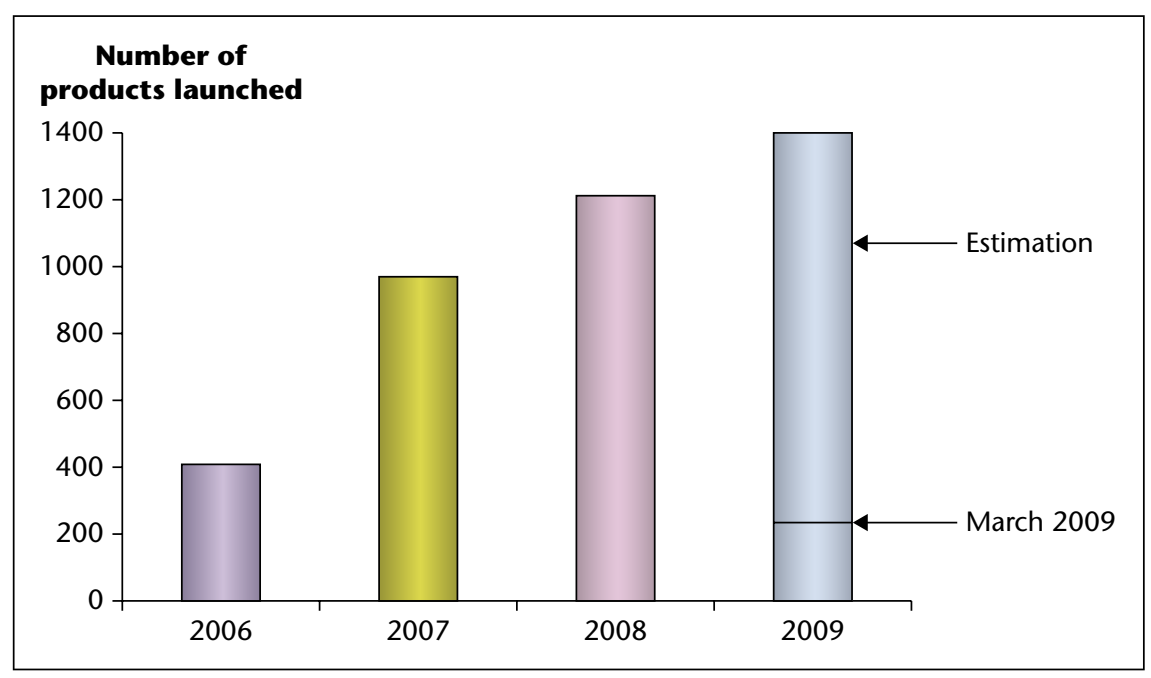

Figure 1. Number of product launches with butters in Europe.

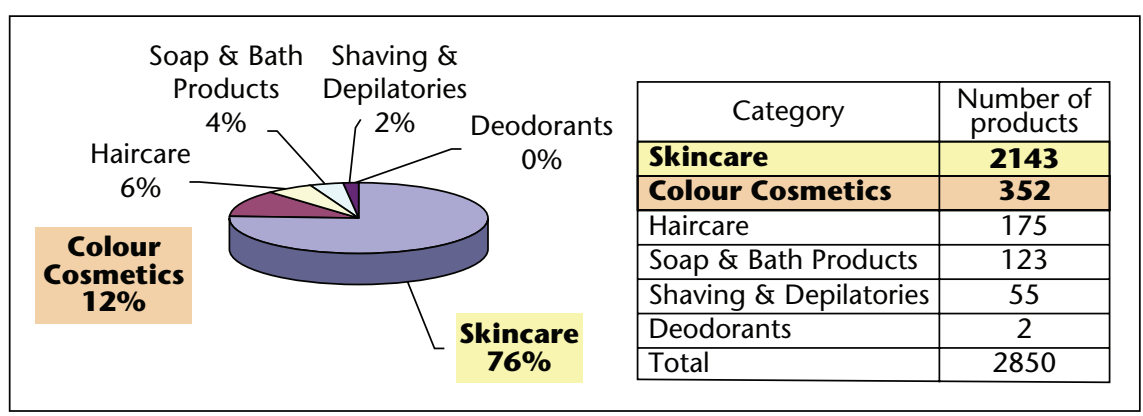

Figure 2. Product categories. 
éviter de refroidir les produits trop rapidement. En effet, des acides gras de faibles poids moléculaires peuvent alors précipiter et casser l'émulsion. La dose d'utilisation peut varier de 2 à $90 \%$.

\section{Propriétés des beurres en cosmétique}

Les beurres sont généralement utilisés pour obtenir des textures originales qui peuvent être souples ou dures selon l'ingrédient utilisé. Les propriétés visées sont aussi l'amélioration du toucher, de l'étalement, l'épaississement des produits et l'émollience Certains beurres sont formulés afin de bénéficier d'un parfum naturel. C'est le cas du beurre de cacao. Les beurres ont la capacité de renforcer le film lipidique présent à la surface de la peau (figure 4). Ils nourrissent et hydratent la peau. Finalement, selon leur indice de réfraction, les beurres peuvent contribuer à améliorer la brillance des produits sur la peau. Dans la partie « insaponifiable» présente dans la plupart des beurres, on trouve généralement une grande quantité d'antioxydants. Ceux-ci peuvent contribuer à améliorer la stabilité des produits et à protéger la peau contre les radicaux libres responsables du vieillissement cutané.

\section{Évaluation sensorielle des beurres [1]}

II est important de réaliser des analyses sensorielles même avec des panels limités de personnes pour caractériser les beurres. Le beurre de cacao donne l'impression d'un étalement rapide, et un effet dur et gras sur la peau. Le beurre de Cupuaçu est plus « long » à l'étalement et donne une impression « crémeuse et riche » sur la peau (figure 5).

Ces propriétés sensorielles sont généralement reliées à la composition. Le beurre extrait du Kokum possède une quantité importante de dérivés de l'acide stéarique dont la chaîne grasse est longue et saturée, ce qui donne une structure moléculaire « organisée » (figure 6). Cet ingrédient a donc un point de fusion relativement élevé et une transformation rapide de l'état de solide à l'état liquide. Le beurre de karité qui possède un taux d'acides gras insaturés important aura un point de fusion plus bas et une transition solide-liquide beaucoup plus longue, conséquence $d^{\prime}$ une structure moléculaire moins organisée.

Grâce à la technique de la RMN, on peut mesurer le taux de solide présent dans un beurre en fonction de la température (figure 7). Le beurre A sera dur dans le produit fini mais liquide sur la peau alors que le $C$ donnera une sensation de dureté car il n'est pas totalement fondu à $37^{\circ} \mathrm{C}$, la température de l'épiderme.

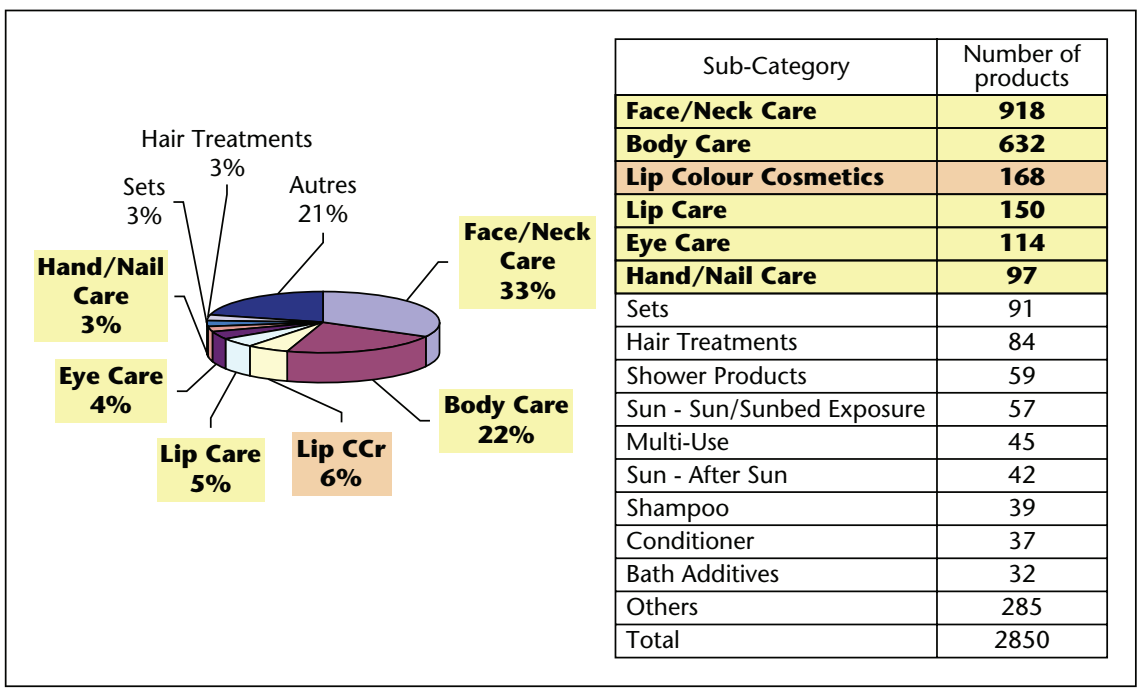

Figure 3. Product sub-categories.

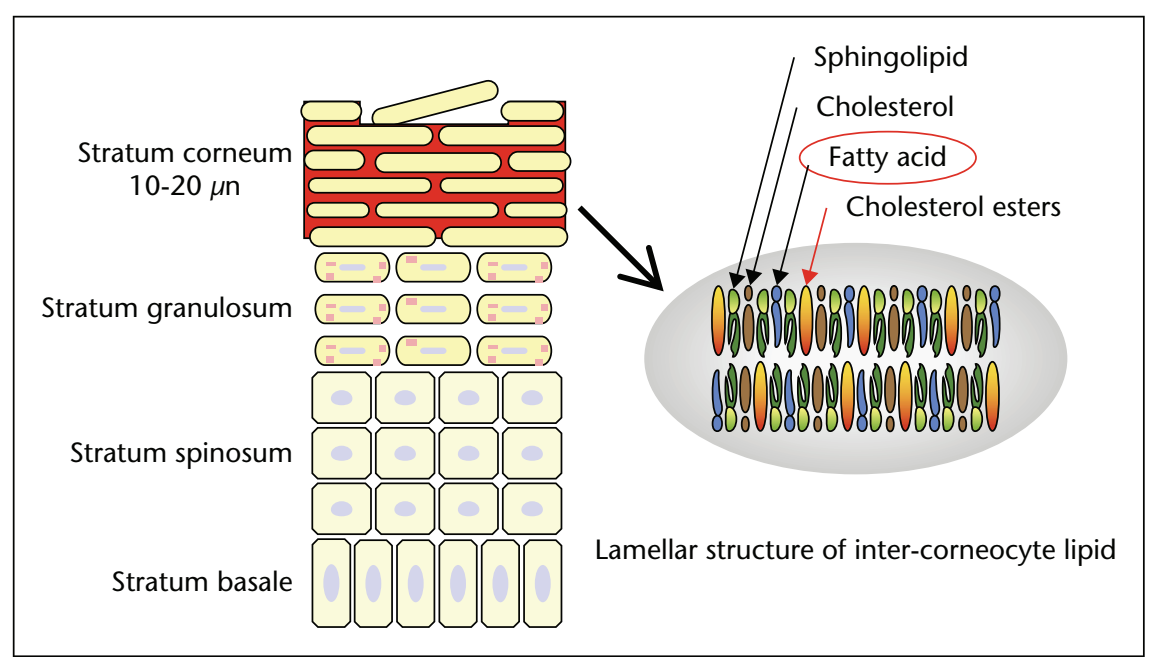

Figure 4. Butters strengthen the inter-corneocyte lipid structure.

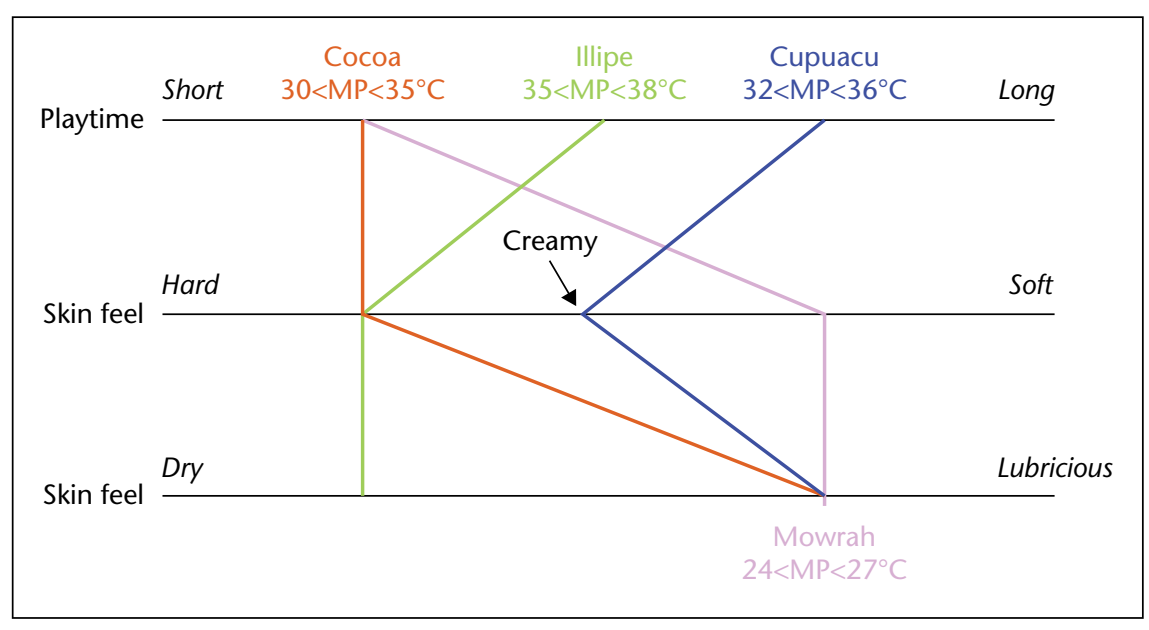

Figure 5. Sensorial evaluation. 


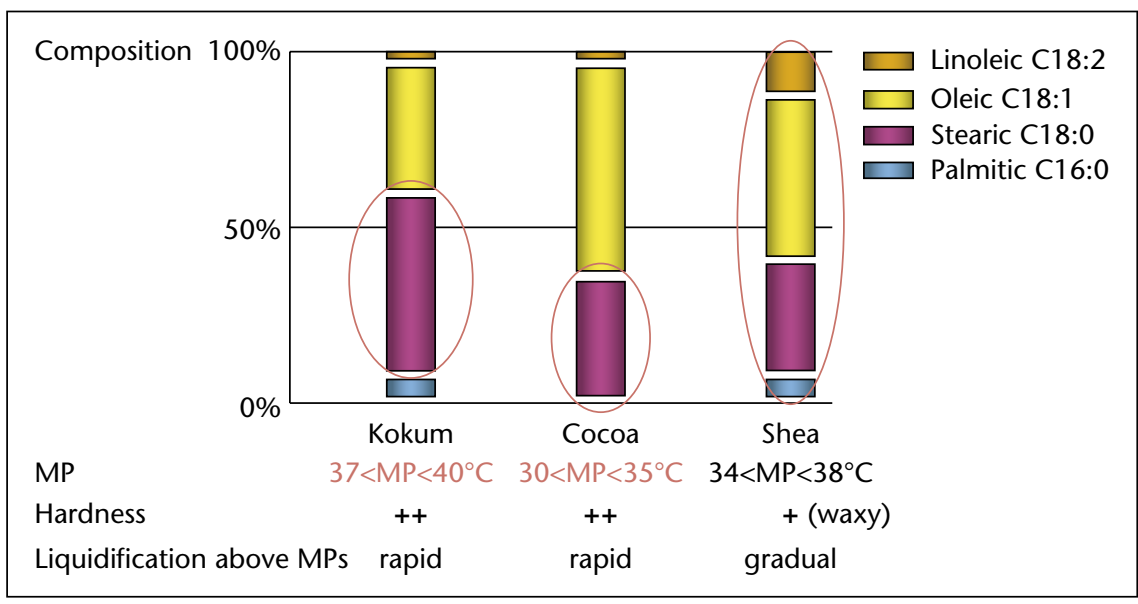

Figure 6. Sensorial properties are linked to fatty acid composition.

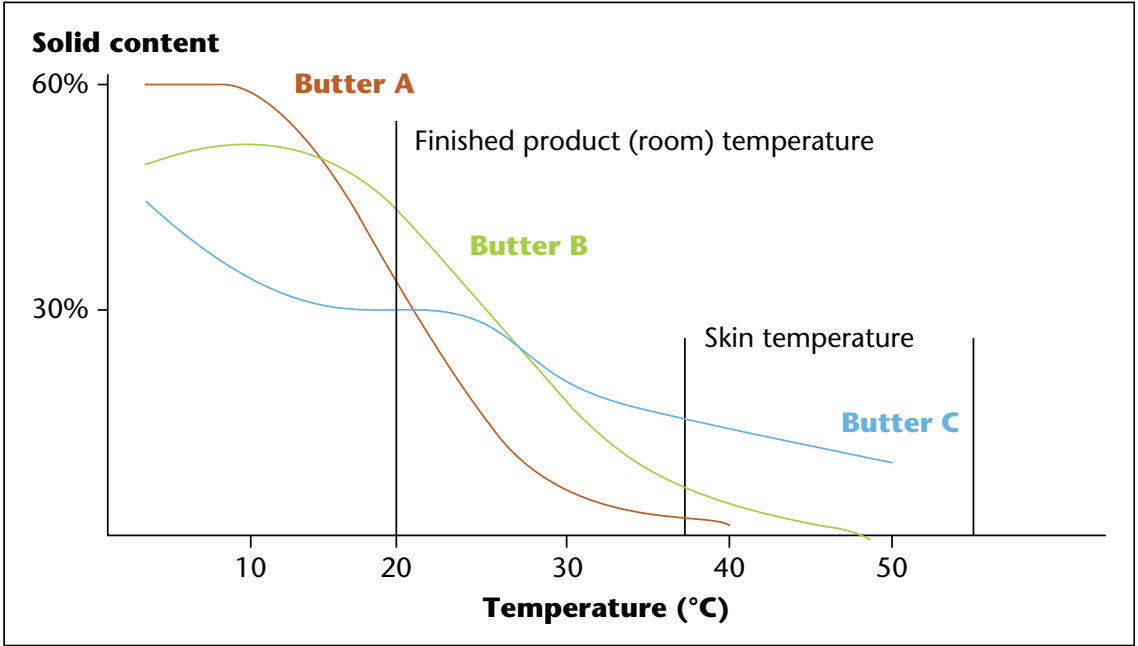

Figure 7. Solid contents can be measured as a function of temperature by NMR.

\section{Les beurres comme outil marketing [2]}

Chaque beurre possède une histoire qui peut servir de base à un argumentaire marketing. Par exemple, le beurre d'Illipe que les Dayaks sur l'île de Bornéo appelaient « l'arbre de jeunesse » servait à préparer des pommades pour le corps. Les Pipils, une civilisation précolombienne, enduisait le corps des adolescents de beurre de cacao pour les préparer au passage à l'état d'adulte.

\section{Conclusion}

Les beurres naturels s'inscrivent parfaitement dans la tendance pour les produits naturels, exotiques et dérivés du secteur alimentaire. C'est pourquoi il y a de plus en plus de lancements de produits cosmétiques en Europe avec des beurres naturels que l'on trouve principalement dans les produits de soin et de maquillage. Les beurres répondent à de nombreuses fonctionnalités importantes en cosmétiques: douceur, hydratation, parfumage des produits. Il est important d'étudier la structure des beurres pour comprendre et anticiper leurs propriétés. Finalement, ce sont des outils marketing très efficaces, un critère particulièrement important dans le domaine des cosmétiques.

\section{RÉFÉRENCES}

1. Moroni LS. Formulating with exotic butters. Personal Care, March 2008, 13-16

2. Dweck AC. Natural Butters. Personal Care Europe, March 2009. 